\title{
Assessment of the Efficiency of the Ghana National Health Insurance Using Network Analysis
}

\author{
Wu Jiying, Jean-Jacques Dominique Beraud, and Evans Kwabena Tekyi Ankomah-Asare
}

\begin{abstract}
This study sought to assess the efficiency of the National Health Insurance Scheme (NHIS) in Ghana. Data was obtained from the National Health Insurance Authority (NHIA) website in Ghana. We divided the NHIS into four main zones (Northern, Southern, Eastern and Western zone) and grouped per the number of facilities under the NHIA (hospital, clinic, pharmacy, government disbursement) weighted against the number of referrals, the number of professional staffs and funding. Network analysis and regression methodology was used to study and analyze the data. Our result showed that the number of professional staff and funding are insignificant in some zones. However, the overall analysis shows that the number of professional staff has a positive influence on the NHIA network; there is a positive flow of information within the network, which facilitate the number of referrals within the network. Funding is insignificant because the first aim of the NHIA is to meet subscribers' satisfaction and alleviate the cost of treatment. Efficiency can be improved by establishing a system that validates and controls the bodies under the NHIA.
\end{abstract}

Key Words-Efficiency, National Health Insurance Scheme, Network.

\section{INTRODUCTION}

Insurance is a system put in place to allow interested people to invest periodically (premiums) into a central domain called "pool" purposely used to compensate individuals who suffer from a loss for which contributions were made. According to [1] in his article, Malaysia's Finance Minister Lim Guan Eng announced that from $1^{\text {st }}$ January 2019 , there would be free national insurance for the low income. He stated that "this scheme is a significant step taken by the Pakatan Harapan (PH) Government to establish a comprehensive social safety net from the aspect of inclusive health insurance and takaful (syariah compliant) coverage for low-income groups for free."

The establishment of the National Health Insurance Scheme (NHIS) in Ghana was an ideology established by former president John Kufuor. He promised to abolish the cash and carry system of health delivery during the 2000 presidential election's campaign. Under the cash and carry system, patients were only treated after an initial payment for the service provided. Even in cases when patients had been brought into the hospital on emergencies, it was required that money was paid at every point of service delivery.

Former president Kufuor through his idea was able to get rid of "cash and carry" and replacing it with an equitable insurance scheme that ensured that treatment was provided first before payment for Ghanaian citizens. In 2003, the scheme was voted and passed into law. Under the law, there was the establishment of Ghana National Health Insurance Authority (NHIA), which licenses, monitors and regulates the operation of health insurance schemes in Ghana. This health insurance was designed to meet specific needs of Ghanaian citizens. The National Health Insurance Scheme (NHIS) is a form of National health insurance established by the Government of Ghana. The scheme provides equitable access and financial coverage for basic health care services to the Ghanaian population. The objective of the NHIS is to secure the implementation of the national health insurance policy that ensures access to basic healthcare services to all residents of Ghana.

Three types of health insurance schemes were put in place, they were: The District-Wide Mutual Health Insurance Scheme, the Private Mutual Health Insurance Scheme and the Private Commercial Health Insurance Scheme.

In order for the system to function well, the government decided to support the District Mutual Health Insurance Scheme concept to ensure that opportunity is provided for all Ghanaian citizens to have equal access to the functional structures of health insurance. Citizens do not move from an unaffordable 'Cash and carry' regime to another unaffordable Health Insurance one. A sustainable Health Insurance option was made available to all Ghanaian citizens and the quality of health care provision is not compromised under Health Insurance. Since the inception of the scheme in 2003, there have been many controversies surrounding its operation and purpose. The first one was to do with members of the opposition National Democratic Congress (NDC), whose members claimed that the scheme was one made for members of the then ruling New Patriotic Party (NPP) [23]. As such, many members of NDC did not want to register with the scheme. The reverse of all the propaganda that surrounded the scheme at its inception were revisited in 
2009 when the NDC took over power. Currently, the controversy with the scheme has to do with the proposed one-time premium payment. This idea was included in the ruling party's manifesto. The promise has so far not come into fruition as the date for its implementation is constantly postponed. Many critics of the proposal claim that it is just not possible to support the scheme with a one-time premium since the sustainability of the scheme would not be possible if premiums were not paid yearly [20].

\section{LITERATURE REVIEW}

A mechanism for addressing financial access constraints affecting mainly the poor posed by the "user-fee" system was substitute by the National Health Insurance Act 605 in 2003 by the Government of Ghana; it imposed the establishment of district-wide Mutual Health Organizations (MHO) [22]. According to [1], there is a global concern regarding how households could be protected from relatively large healthcare payments that are a major limitation to accessing healthcare. Such payments also endanger the welfare of households with the potential of moving households into extreme impoverishment. In their paper, they paper studied the impoverishing effects of out-of-pocket (OOP) healthcare payments in Ghana prior to the introduction of Ghana's national health insurance scheme. Poverty lines $(\$ 1.25$ and $\$ 2.50$ per capita per day at the 2005 purchasing power parity) are used in assessing the impoverishing effects of OOP healthcare payments. Evidence revealed that there was a high incidence and intensity of impoverishment due to OOP healthcare payments in Ghana due to the absence of financial risk protection. [2] in their paper investigated if health insurance can protect against out-of-pocket and catastrophic expenditures and support poverty reduction. They analyze the effect of health insurance on household out-of pocket expenditure (OOPE), catastrophic expenditure (CE) and poverty. They employed probit models and use of instrumental variables to analyze the effect of health insurance on OPE, CE and poverty. Their study revealed that health insurance enrolment reduced OOPE by $86 \%$ and protected households against CE and poverty by $3 \%$ and $7.5 \%$ respectively, which support the pro-poor policy objective of Ghana's National Health Insurance Scheme and holds relevance to other low and middle-income countries implementing or aiming to implement insurance schemes [24].

[3] in their study about administrative Efficiency and policy failure proposed some theories to evaluate the determinants of Administrative failure to ensure policy efficiency for health insurance. They did a comparison between healthcare providers and insured person based on some questionnaire. Their study revealed that the NHIS is efficient in a way but its administrative failure was what entangles controversies in the field of debate.
It was revealed that the administrative failure was caused by several factors including delays in reimbursing healthcare providers, insured patients were charged relative additional cost for drugs. They suggested that policy makers should take action to restrain administrative failures when implementing policies. [4]in their study explored the efficiency levels of NHISaccredited private and public health facilities in order to ascertain factors that account for differences in efficiency and determine the association between quality care and efficiency levels. They used a cross-sectional survey of NHIS-accredited primary health facilities in two regions in southern Ghana. Data Envelopment Analysis was used to estimate technical efficiency of sampled health facilities while Tobit regression was employed to predict factors associated with efficiency levels. Spearman correlation test was performed to determine the association between quality care and efficiency. Their findings recommended some level pf wastage of health resources in many healthcare facilities especially those located in urban areas. [5] examined the equity in coverage under Ghana's National Health Insurance Scheme. They used bivariate and multivariate methods to analyze a survey composed by both males and females samples respectively, concentration curves and indices were used to examine equity in coverage on the NHIS. It revealed that coverage was highest among the highly educated, professionals, those from households in the richest wealth quintile and urban residents. Lack of coverage was most concentrated among the poor.

[6] examined the association between health insurance status and utilization of outpatient and inpatient health services in rural poor communities. The study was based on a cross-sectional household survey conducted in the Kassena-Nankana districts of Northern Ghana. They used multiple logistic regression models to identify factors associated with the utilization of outpatient and inpatient health services. The dependent variables were the utilization of outpatient and inpatient health services. We adjusted for several potential socio-demographic factors associated with utilization and health insurance status. They suggested that being insured with the NHIS is associated with increased utilizations of outpatient and inpatient health services in the studied area. [7, 8]compared health and economic indicators, described the structure of each country's NHIS within the wider healthcare system, and analyzed it impacts on equity in financing and access to health care. They used the World Bank and other sources to elaborate a comparative and economic data. It revealed that major health indicators are more favorable in Ghana and overall equity in financing and access are weaker in Nigeria.

[8] examined the determinants of National Health Insurance (NHIS) enrolment in Ghana, using two different surveys and distinguishing between younger and older adults. The comparison between age groups 
was aimed at understanding whether determinants differ for older adults. They used a logistic regression was used to explored the socio-economic and demographic determinants of NHIS enrolment and multinomial logistic regression investigated the correlates of insurance drop out. The paper indicated that although the gap in coverage among rich and poor, urban, and rural residents appears to have decreased, these factors still determine NHIS coverage of younger and older adults. [9] investigated the impact of Ghana's National health insurance scheme median based on pharmaceutical pricing methodology and reimbursement policy on the pharmaceutical system. Quantitative medicine claims data for the period were also collected to analyze patterns of medicine utilization and reimbursement values. Market dynamics, foreign exchange and medicine prices were considered in the comparison of reimbursement prices with the market value. They suggested that it is critical to consider medicines in all conversations, design and planning of programs due to their clinical and financial impact.

\section{METHODOLOGY}

We used a social network or network Theories, which will help in optimizing the prediction of future Health Insurance claims to aid government budgeting and policy makers. A complex model was used to group various hospitals under the NHIA per their weight or contribution to the National Health Insurance. The importance of claim source: a success on a multi-attribute decision or characteristics.it will help in the determination of specifics claims sources as a mean of defining their weight in the claim network. The consideration on variables: weight determinant will be the average patient treated at time $t$, the number of Health staff (doctors, nurses, pharmacist and others (administrators)). Pharmaceutical dispense (Average cost of pharmaceutical dispensation). Utility cost (electricity, water, and others) assumed to be incurred by the hospital trough patients or visitors. The data collected is a secondary data from ministry of Health database.

We then conducted a regression analysis of the network output as a mean of determining the generic coefficient of claim. Neural prediction to determine the best optimum sealing to sustain the Health Insurance policy. We selected in all 200 hospitals each from each region. In 2017, The National Health Insurance Authority recorded over 400 hospitals under its jurisdiction. The selection consisted of the top 10 hospitals with the highest claims per district, per region under the National Health Insurance Authority and the lowest 10 hospitals claims per district. We divided the bodies (Pharmacies, clinics, hospitals and government disbursements) into four main zones: Northern zone, Southern zone, Eastern zone and Western zone.

The sum of the Hospitals $\sum_{i=1}^{m} b_{i}$ will determine the weight of each claim source (Hospitals). We will use the $\log$ of the sum to normalize the weights and then plot the network.

In all these, distance between the weights will not be factored; it will be assumed that they are all operating in Ghana (Euclidian space). To normalize the distance between nodes, we will apply the Kamada-Kawai [29] spring embedder model to our NHIA network, thus allowing us to consider hospitals networks to be dynamic systems where every edge between two hospitals is a "spring" of a desirable length, which corresponds to their graph theoretical distance. In this way, the optimal layout of the graph is the state with the minimum imbalance. The degree of imbalance is formulated as the total spring energy; the square summation of the difference between desirable distances and real ones for all pairs of nodes. We will analyze the network base on Centrality test, Clustering and density

The degree centrality is a function of a node's number of edges. It reflects the node's communication potential within the network. For our thesis, a node's Degree centrality is defined as $\sum_{j \neq i} a_{i j}$ where $a_{i j}$, represent the different component of each group (cluster).

The Normalization of the decision matrix is done using the below transformation for each node $n_{i j}: n_{i j}=$ $\frac{a_{i j}}{\sqrt{\sum_{i=1}^{m} a_{i j}^{2}}}, a_{i j}$ various component of each Cluster. Then, weights should be multiplied to normalized matrix. Decision makers need to reach the level of the consistency of judgments, because inconsistent judgments may lead to meaningless decisions. Saaty (1980) proposed the formula for calculating inconsistency: $C I_{n}=\frac{\lambda_{\max }-n}{n-1} \mathrm{n}$ represent the number of comparison (number of cluster), $\lambda_{\max }$ represent the maximal Eigenvalue and $\lambda_{\max }>n$

$A x=\lambda_{\max } x$, where $\mathrm{A}$ is the comparison matrix of size $\mathrm{n}^{*} \mathrm{n}$, for $\mathrm{n}$ criteria, and $\mathrm{x}$ is the Eigenvector of size $\mathrm{n}^{*} 1$.

Let $y_{i}$ denote the number of claims per group, $X_{i 1}$ represents the number of patient treated at the hospital, $X_{i 2}$ represents the management size, $X_{i 3}$ represents the professional size (doctors, nurses) and $X_{i 4}$ represents the ownership type (private and government) our regression model will be define as

$y_{i}=\beta_{o}+\beta_{1} X_{i 1}+\beta_{2} X_{i 2}+\beta_{3} X_{i 3}+\beta_{4} X_{i 4}+\varepsilon_{i}$

The general regression model for the NHIA network will be defined as:

$Y=\beta_{o}+\beta_{1} X_{1}+\beta_{2} X_{2}+\beta_{3} X_{3}+\beta_{4} X_{4}+\varepsilon_{j}$

Where $\mathrm{Y}$ represent the total number of claims, $X_{1}$ represent the total number of patient treated at the various hospital under the NHIA, $X_{2}$ represent the overall management size, $X_{3}$ total number of doctors and nurses, $X_{4}$ total number of ownership type.

Where i represent the number of cluster $i=1,2,3 \ldots, n$ 


\section{DATA ANALYSIS \\ A. Trend analysis}

The group K-step reach is the number or proportion of non-group members that can be reached from a group member in K steps or less. Directed versions of both these measures can be constructed in the same was as for individual actor centralities. This routine takes an actor by group indicator matrix and calculates these measures for each group given in the columns of the matrix.
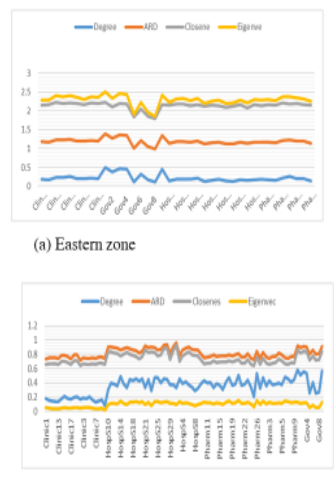

(c) Southern Zone
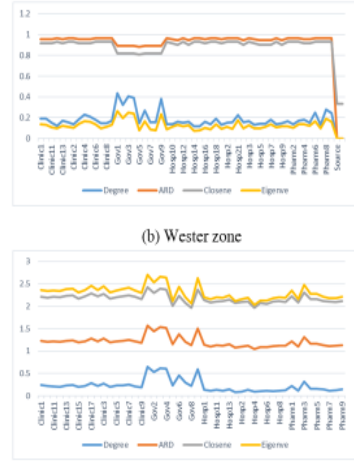

(d) Northern Zone
Fig. 1. Group centrality plot of the NHIA network

From Figure 1 (a), we can see that the closeness and Eigen vectors are walking along it means that there is a strong relation or connection within the network. The Eigen vector represents the value of the connection of an actor within the network, with respect to other actors within the same network. We can see that the degree of centrality of the whole network lies between 0-0.5. ARD represents the Average Reciprocal distance between actors within the network. From (b) we can see that the Eigenvector within the network lie between 0 and 0.3, which means that there, is no outstanding actor having more importance than the other within the network. The degree centrality lies between 0.1 and 0.5 . (c) Shows that the Eigenvector within the network lie between 0 and 0.2, which means that there, is no outstanding actor having more importance than the other within the network. The degree centrality lies between 0.2 and 0.6 . Both the ARD and closeness lie between 0.6 and 1. (d) Reflect that both the Eigenvector and closeness within the network lie between 2 and 3, which means that there are outstanding actors having more importance than others within the network. The degree centrality lies between 0.2 and 0.6 . The ARD lies between 1 and 1.6.

\section{B. Centrality analysis of the network}

The group degree centrality of a group of actors is the size of the set of actors who are directly connected to group members. This can be normalized by considering the size of the non-group member set.
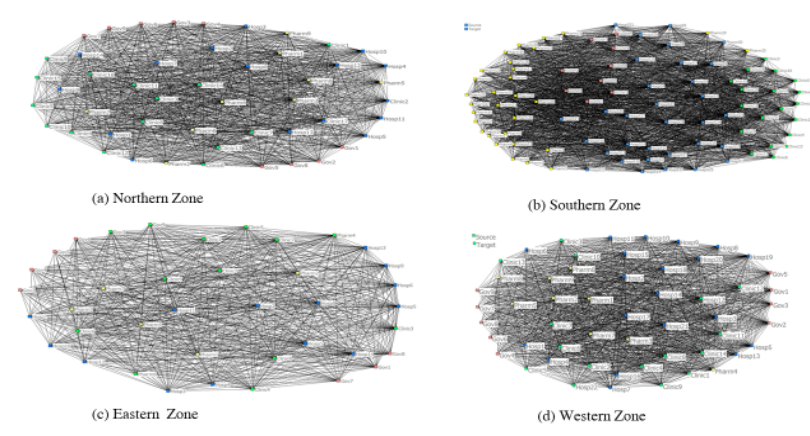

Fig. 2. Overview of the zonal NHIA's network

Figure 2 displays how the various body or actors of the NHIA zone are connected to each other. We have nine periodic sources of funding of the NHIA, which are:

- The National Health Insurance Levy (NHIL) which is a 2.5 percent value added tax (VAT) levied on selected goods and services.

- 2.5 percent social security deductions from formal sector workers managed by the Social Security and National Insurance Trust (SSNIT)

- GOG annual budgetary allocations proposed and approved by parliament to the NHIF.

- Accruals from investment of surplus funds held in the NHIF by the National Health Insurance Council (NHIC)

- Premiums/ contributions paid by NHIS subscribers

- Donation by NGos (UNICEF, PNUD, UN)

- District Assembly Fund

- Sub-District Assembly Fund

- Regional Fund allocated to sustain and improve healthcare conditions

The Northern network displays in total, 14 hospitals (Hosp), 17 clinics, 9 pharmacies (Pharm), and 9 sources (Gov) of funding under the NHIA. Each actor is connected to more than one node. In this network, we have some actors that carry more weight since they have more connection to other nodes. The Southern zone displays in total, 31 hospitals (Hosp), 19 clinics, 31 pharmacies (Pharm), and 9 sources (Gov) of funding under the NHIA. Each actor is connected to more than one node. In this network, we have some actors that carry more weight since they have more connection to other nodes. We can see that some actors carry more weight, which is they have more connection than others within the network do. (For example Hosp 29,24,3, Clinic10, 1, 16, Pharm 26, 1, 2 and so on). The Easter zone displays in total, 13 hospitals (Hosp), 8 clinics, 7 pharmacies (Pharm), and 9 sources (Gov) of funding under the NHIA. Each actor is connected to more than one node. In this network, we have some actors that carry more weight since they have more connection to other nodes. We can 
see that some actors carry more weight, which is they have more connection than others within the network do. The Western network displays in total, 21 hospitals (Hosp), 19 clinics, 9 pharmacies (Pharm), and 9 sources (Gov) of funding under the NHIA. Each actor is connected to more than one node. In this network, we have some actors that carry more weight since they have more connection to other nodes. We can see that some actors carry more weight, which is they have more connection than others within the network do.

\section{General Statistics of the NHIA network} This part deals with the general overview of the NHIA network per zone. Table 1 represent the general test statistics of the NHIA based on information flow, eigenvector, referrals and freeman closeness average.(Insert Table 1)

We can identify the significant variables within the network. Hence, we can identify that per the number of professional, the information flow is significant for only the Northern Zone and Southern zone. The freeman Closeness Average is significant for eastern and the northern zone. In addition, the number of referrals per the number of professional within the whole network is significant for the Eastern, Western and Northern Zone. However, in terms of funding, we realized that it has no influence on the network; it means there is no major impact of funding within the network.

\section{FACTORS INFLUENCING THE EFFICIENCY OF THE NHIS CLAIMS EXPENDITURE}

The size and efficiency of the Ghana national claims expenditure are determined by three Factors: coverage expansion, behaviors of service providers and National Health Insurance Scheme (NHIS) members,

and the internal management of the National Health Insurance Authority (NHIA). Insurance seeks to remove financial barrier for accessing care by expanding coverage. Policymakers' ability to influence this dimension is inherently limited. However, the authorities can influence the behavior of service providers and patients through measures to address adverse selection during enrollment, the suboptimal composition of the benefits package, low levels of cost-consciousness, and weak performance incentives. The NHIA can also improve its own internal efficiency by reforming its systems for claims processing, provider oversight, and member engagement.

The central government manages salaries and allowances for workers in public facilities, and payment is not linked to either output or outcome indicators. NHIS provider payments are based on vetting reports and do not consider value of money. There is no mechanism to identify, publicize, or reward individuals and facilities that provide high-quality services or reduce health care costs. NHIS accreditation focuses on the quality of a facility's human resources, supplies, equipment, and infrastructure, but it does not directly capture service quality.

Claims processing by NHIA is labor-intensive and inefficient. Claims are vetted on an individual basis. Most claims are evaluated manually, even the relatively small share that are electronically submitted. The NHIA expends staggering 1,200-5,000 staff weeks vetting each month's claims, and maintaining this schedule requires hundreds of staff members (Table 2).

No estimates are available on the quality of claims vetting at the national level, but an analysis of claims in an area of the Southern zone suggested that the process is subject to significant errors. The claims data of that area revealed that 18 percent of submitted claims lack essential information or are submitted by unaccredited facilities under the NHIA. These claims must either be rejected or sent back to the facility for correction. Unfulfilled claims represent just 3 percent of the value of all claims in Volta.

Previous claims-expenditure reviews have shown that some service providers display abnormal behavior that can indicate fraud or abuse and that warrants additional scrutiny. Private facilities, which tend to be high-cost providers, are more likely than other facilities to submit incomplete claims information. Moreover, among private clinics that submit claims without GDRG information, 42 percent also lack diagnosis information, making it impossible to determine whether the GDRG is appropriate and these claims expenditure are eligible for reimbursement. NHIA's claims-vetting system is not properly equipped to identify abnormal behavior among service providers. Claims offer a wealth of information on expenditure patterns, but most of the data captured by NHIA are not analyzed [28]. (Insert Table2)

\section{CONCLUSION AND RECOMMENDATION}

The network analysis revealed that there is a dysfunction of the NHIS within the four zone. This is due to the inefficacity of the NHIA to fully control the facilities under its jurisdiction. The study also revealed that both the southern and the northern zone have more influence than the other zone. They carry more weight (funding, professional staff) than the others do. The deficit of the NHIA is due mainly due to inability of controlling the claims submitted by various bodies, because they use a manual system to check each claims submitted, leading to the inflation of claims, long period of auditing and delay in payment. We suggest that NHIS establish a 
stronger expenditure control system for long-term sustainability. The majority of NHIS claims expenditures is mainly composed of outpatient consultations and district hospitals. These distribution patterns are related to NHIS design features that encourage expenditure surges. The claims processing system should have computerized so that it can collect information on service delivery and results. Mechanisms should be adopted to monitor and correct providers' abnormal behaviors, or to engage NHIS members for information verification, case management, and prevention.

\section{A. Recommendation}

The enrollment process should be redefined to address adverse selection because the NHIS is mainly financed by taxes. It should not attempt to omit high-cost individuals in order to address adverse selection. However, the NHIS must provide incentives for low-cost individuals to enroll and remain within the scheme.

The NHIA should improve its benefits package. The international standard norm suggests the importance of adopting a positive list of included benefits rather than a list of excluded conditions, then gradually expand the benefits package to manage cost increases and implementation challenges, and establishing a straightforward institutional process for adjusting the benefits plan.

Reimbursement mechanisms must be reframed in such a way that it can increase providers' cost consciousness and maximize value for money. Expending the treatment areas to which capitation payments apply (e.g., pharmaceutical prescriptions, inpatient services) would discourage providers from shifting financial risks to feefor-service. Policymakers must take steps to ensure that these measures do not prompt service providers to increase financial pressure on patients.

\section{B. Further Studies}

The NHIA should gather information that is more comprehensive on interactions between patients and health care providers. Apart from the basic information needed to process claims, the NHIA should collect patients' health status that is, any tests undertaken, any procedures performed, the results of lab work, any medicines prescribed, and the total costs incurred. This information will help the NHIA to judge the aptness of diagnoses, claims elaboration, and treatment plans. It will help the NHIA avoiding double payment and specific diagnosis cost while paying providers.

\section{ACKNOWLEDGEMENT}

This study was funded by The Research Youth Fund Project of Ministry of Education of China with Grant number 17YJC910008.

\section{REFERENCE}

[1] Akazili, J., et al., Assessing the impoverishment effects of outof-pocket healthcare payments prior to the uptake of the national health insurance scheme in Ghana. BMC International Health and Human Rights, 2017. 17(1): p. 13.

[2] Aryeetey, G.C., et al., Can health insurance protect against out-of-pocket and catastrophic expenditures and also support poverty reduction? Evidence from Ghana's National Health Insurance Scheme. International Journal for Equity in Health, 2016. 15(1): p. 116.

[3] Sanyare, F.N. and J.G. Tuolong, Administrative Efficiency and Policy Failure: The National Health InsuranceGlobal Encyclopedia of Public Administration, Public Policy, and Governance, A. Farazmand, Editor. 2017, Springer International Publishing: Cham. p. 1-9.

[4] Alhassan, R.K., et al., Efficiency of private and public primary health facilities accredited by the National Health Insurance Authority in Ghana. Cost Effectiveness and Resource Allocation, 2015. 13(1): p. 23.

[5] Dake, F.A.A., Examining equity in health insurance coverage: an analysis of Ghana's National Health Insurance Scheme. International Journal for Equity in Health, 2018. 17(1): p. 85.

[6] Dalinjong, P.A., et al., The association between health insurance status and utilization of health services in rural Northern Ghana: evidence from the introduction of the National Health Insurance Scheme. Journal of Health, Population and Nutrition, 2017. 36(1): p. 42.

[7] Odeyemi, I. and J. Nixon, Assessing equity in health care through the national health insurance schemes of Nigeria and Ghana: a review-based comparative analysis. International Journal for Equity in Health, 2013. 12(1): p. 9.

[8] Van der Wielen, N., J. Falkingham, and A.A. Channon, Determinants of National Health Insurance enrolment in Ghana across the life course: Are the results consistent between surveys? International Journal for Equity in Health, 2018. 17(1): p. 49

[9] Mordi, D.O., K. Eghan, and J. Rankin, The impact of Ghana's National Health Insurance Scheme median pharmaceutical pricing methodology and reimbursement policy on the pharmaceutical system. Journal of Pharmaceutical Policy and Practice, 2015. 8(1): p. P5.

[10] Cordero, J. M., Alonso-Morán, E., Nuño-Solinis, R., Orueta, J. F., \& Arce, R. S. J. E. J. o. O. R. (2015). Efficiency assessment of primary care providers: A conditional nonparametric approach. 240(1), 235-244.

[11] Dake, F. A. A. (2018). Examining equity in health insurance coverage: an analysis of Ghana's National Health Insurance Scheme. International Journal for Equity in Health, 17(1), 85. doi:10.1186/s12939-018-0793-1

[12] Dalinjong, P. A., Welaga, P., Akazili, J., Kwarteng, A., Bangha, M., Oduro, A., . . Goudge, J. (2017). The association between health insurance status and utilization of health services in rural Northern Ghana: evidence from the introduction of the National Health Insurance Scheme. Journal of Health, Population and Nutrition, 36(1), 42. doi:10.1186/s41043-017-0128-7

[13] Debpuur, C., Dalaba, M. A., Chatio, S., Adjuik, M., \& Akweongo, P. (2015). An exploration of moral hazard behaviors under the national health insurance scheme in Northern Ghana: a qualitative study. BMC Health Services Research, 15(1), 469. doi:10.1186/s12913-015-1133-4

[14] Dictionary, C. (Ed.) (2019) CambridgeDictionary. London: Cambridge

[15] Duku, S. K. O. (2018). Differences in the determinants of health insurance enrolment among working-age adults in two regions in Ghana. BMC Health Services Research, 18(1), 384. doi:10.1186/s12913-018-3192-9

[16] Fatunde, O. A., \& Bhatia, S. K. (2012). Case Study of Ghana. 
In Medical Devices and Biomaterials for the Developing World: Case Studies in Ghana and Nicaragua (pp. 19-32). New York, NY: Springer New York.

[17] Ghafoor, F., \& Niazi, M. A. (2016). Using social network analysis of human aspects for online social network software: a design methodology. Complex Adaptive Systems Modeling, 4(1), 14. doi:10.1186/s40294-016-0024-9

[18] Ghana. (2003). National health insurance scheme. Ghana Retrieved from http://www.nhis.gov.gh/

[19] Health, T. I. o. P. (2018). Health Care and Social Services.

[20] Hsu, J. C., \& Lu, C. Y. (2015). The evolution of Taiwan's National Health Insurance drug reimbursement scheme. DARU Journal of Pharmaceutical Sciences, 23(1), 15. doi:10.1186/s40199-014-0080-7

[21] Inkoom, D. K. B., \& Gyapong, A. Y. (2016). Decentralization in Africa: Local Government and Health Care in Ghana, Malawi and Tanzania. In C. N. Silva (Ed.), Governing Urban Africa (pp. 99-124). London: Palgrave Macmillan UK.

[22] McGihon, R., Hawke, L. D., Chaim, G., \& Henderson, J. (2018). Cross-sectoral integration in youth-focused health and social services in Canada: a social network analysis. BMC Health Services Research, 18(1), 901. doi:10.1186/s12913018-3742-1

[23] Mordi, D. O., Eghan, K., \& Rankin, J. (2015). The impact of Ghana's National Health Insurance Scheme median pharmaceutical pricing methodology and reimbursement policy on the pharmaceutical system. Journal of Pharmaceutical Policy and Practice, 8(1), P5. doi:10.1186/2052-3211-8-s1-p5

[24] Nsiah-Boateng, E., Asenso-Boadi, F., Andoh-Adjei, F.-X., \& Aikins, M. (2018). Knowledge and satisfaction of health insurance clients: a cross-sectional study in a tertiary hospital in Ghana. Journal of Public Health. doi:10.1007/s10389-018$1000-\mathrm{y}$

[25] Odeyemi, I., \& Nixon, J. (2013). Assessing equity in health care through the national health insurance schemes of Nigeria and Ghana: a review-based comparative analysis. International Journal for Equity in Health, 12(1), 9. doi:10.1186/1475-9276$12-9$

[26] Owoo, N. S., \& Lambon-Quayefio, M. P. (2013). National health insurance, social influence and antenatal care use in Ghana. Health Economics Review, 3(1), 19. doi:10.1186/2191-1991-3-19

[27] Saeed, B. I. I., Yawson, A. E., Nguah, S., Agyei-Baffour, P., Emmanuel, N., \& Ayesu, E. (2016). Effect of socio-economic factors in utilization of different healthcare services among older adult men and women in Ghana. BMC Health Services Research, 16(1), 390. doi:10.1186/s12913-016-1661-6

[28] Sanyare, F. N., \& Tuolong, J. G. (2017). Administrative Efficiency and Policy Failure: The National Health Insurance Scheme of Ghana in Perspective. In A. Farazmand (Ed.), Global Encyclopedia of Public Administration, Public Policy, and Governance (pp. 1-9). Cham: Springer International Publishing.

[29] Shannon, C. (1948). BA mathematical theory of communication. Bell System Tech. J, 27(3).

[30] Suchman, L. (2018). Accrediting private providers with National Health Insurance to better serve low-income populations in Kenya and Ghana: a qualitative study. International Journal for Equity in Health, 17(1), 179. doi:10.1186/s12939-018-0893-y 


\section{APPENDIX}

TABLE 1 GENERAL SUMMARY OF THE NHIA NETWORK

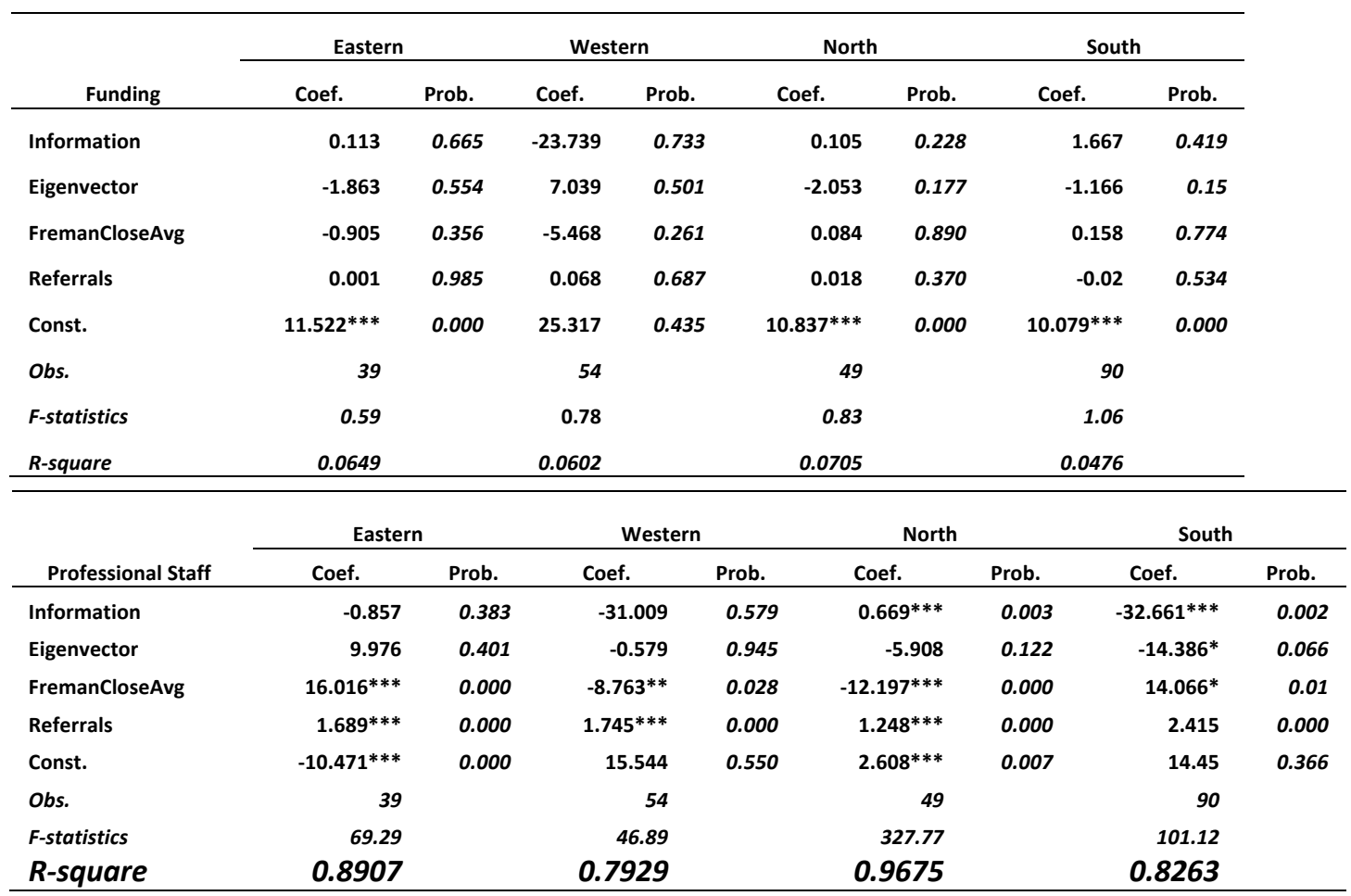

$* * *, * *, *$ indicate significance level at $1 \%, 5 \%$, and $10 \%$ respectively.

TABLE 2 SIMULATION OF LABOR REQUIREMENT FOR PROCESSING ONE MONTH'S NHIS CLAIMS

\begin{tabular}{llc}
\hline $\begin{array}{c}\text { Minutes spent } \\
\text { On each claim }\end{array}$ & $\begin{array}{c}\text { Staff weeks needed } \\
\text { to process each } \\
\text { month's claims }\end{array}$ & $\begin{array}{c}\text { Staff members } \\
\text { needed to proceed } \\
\text { month's claim }\end{array}$ \\
\hline $1^{\text {st }}$ Scenario: One staff member vets 100 claims per day 5.0 & 5000 & 1250 \\
$2^{\text {nd }}$ Scenario: One staff member vets 100 claims per day 2.8 & 2800 & 700 \\
$3^{\text {rd }}$ Scenario: One staff member vets 100 claims per day 1.2 & 1200 & 300 \\
\hline
\end{tabular}

